\title{
Sunlight Illumination Models for Spacecraft Surface Charging
}

\author{
Stuart Grey, Richard Marchand, Marek Ziebart, Roghaiya Omar
}

\begin{abstract}
A model is developed to compute solar illumination of satellite components while accounting for multiple reflections, variable surface properties and illumination source size. This model is implemented in OpenCL and integrated with the particle in cell (PIC) satellite-environment simulation code, PTetra, enabling kinetic simulations in which photoelectron emission resulting from direct illumination and multiple reflections is taken into account. Simulation results obtained with a simple "toy geometry" are used to illustrate the effect of multiple reflections on the net current density at a satellite surface, and on the surrounding space charge potential. Results for the Solar Orbiter spacecraft show a small change to the overall charging and charge distribution around the Solar Wind Analyzer instrument.
\end{abstract}

Index Terms-photoemission, reflectance, spacecraft charging, sunlight charging

\section{INTRODUCTION}

$\mathbf{C}$ HARGING of spacecraft in sunlight has been an important topic of research for many years and is particularly important for modelling the charging during a spacecraft's transition out of eclipse [1], [2]. This is due to the fact that current emitted from spacecraft surfaces often dominates over all other ambient currents in the magnetosphere [3]. The importance of photoemission as a charging process for spacecraft is a result of large photoelectric yields for many materials in the extreme ultraviolet (EUV) range of wavelengths $(<2000$ $\AA$ ) [4], [5] which has been shown by measurement to vary over time [6]. While the accurate determination of the material properties as they relate to photoemission is a known area for improvement [7], little work has been carried out to improve the fundamental illumination models, that is, the model that determines which spacecraft surfaces are illuminated at any given epoch.

In this work we concentrate on improving the modelling of photoemission on the Solar Orbiter spacecraft [8] whose instruments are particularly sensitive to absolute and differential potentials [9].

In order to use the improved illumination model it must be coupled with a numerical simulation of the spacecraft plasma interaction. In this case PTetra[10] was chosen.

Due to the fact that photoemission, like secondary electron emission, is a surface phenomena it is extremely sensitive to changes in surface condition [11] which is known to vary over spacecraft operational lifetimes [12].

S. Grey is with The Department of Mechanical and Aerospace Engineering, University of Strathclyde. e-mail: stuart.grey@strath.ac.uk

R. Marchand and R. Omar are with The University of Alberta.

M. Ziebart is with the Department of Civil, Environmental and Geomatic Engineering, University College London, UK
Analytic models of spacecraft illumination can be developed for simple spacecraft models such as spheres, cylinders and cubes [13] but for the more complex geometry of real spacecraft a different approach must be taken that models the illumination on each of the spacecraft's surfaces.

The photoelectron flux density $J_{p h}(\omega, \alpha)$ emitted from a surface is given by

$$
J_{p h}(\omega, \alpha)=J_{0}(\omega) Y(\omega, \alpha)[1-R(\omega, \alpha)]
$$

where $J_{0}(\omega)$ is the incident light intensity at frequency $\omega$, $Y(\omega, \alpha)$ is the photoelectron yield per absorbed photon, $\alpha$ is the photon incidence angle and $\mathrm{R}$ is the reflectance [14].

In the case of zero reflectance, Eq. 1 implies that the emission of photoelectrons would be maximum, while in the case of perfect reflectance no photoelectrons would be emitted. As a consequence the model suggests that a highly reflective surface will produce very few PE but could in turn reflect a large number of photons onto other surfaces. The exact distribution of the reflected photons is highly dependent on the spacecraft geometry and its attitude in relation to the incoming light source. In this work an illustration of reflection effects is first demonstrated using a simple "toy geometry", followed by an application to the Solar orbiter spacecraft with assumed perfect attitude control.

\section{ILLUMINATION MODELS}

\section{A. Commonly Used Models}

The most common approach when calculating illumination during numerical spacecraft-plasma simulations is to use a ray tracing technique. In computer graphics ray tracing commonly involves propagating rays from the camera into the scene and seeing if either directly, or indirectly (through reflection), they end up at a light source, commonly known as backwards ray tracing.

A similar approach is taken in most spacecraft charging codes such as PTetra[10] and SPIS[15] although a simpler method can be used as only self shadowing is considered in these models. In particular in PTetra the rays originate from each triangular subdivision on the spacecraft model's surface and are propagated towards the light source. If the ray from a given triangular subdivision reaches the lightsource without intersecting any spacecraft geometry then the triangle is fully illuminated, otherwise it is fully in shadow.

This approach is extremely fast to compute and coarsely captures the self-shadowing, but with some limitations. Firstly, 
surface elements are either fully in shadow or fully illuminated, secondly, all incoming rays are often assumed to be parallel, thus not modelling the apparent size of the light source and thirdly, and perhaps most importantly, this approach does not model any reflection of the incoming light onto other surfaces.

The approach demonstrated here solves all of these issues by using a forward ray tracing technique where every ray starts at the light source and is then propagated into simulation space.

\section{B. Forward Ray Tracing}

Forward ray tracing has been successfully used for the computation of extremely accurate models of solar radiation pressure (SRP) on spacecraft [16],[17]. The approach used in this work extends the SRP method by adopting a multisampling approach that enables modelling the apparent size of the illumination source and more accurately modelling diffuse reflection from spacecraft surfaces. A multi-sample technique aims to have a large number of rays striking each element of the spacecraft model. The number of intersections are then counted and the illumination calculated. In this method each ray has its own set of initial conditions and is treated independently, interacting with the spacecraft structure through multiple diffuse and specular reflections as shown in Figure 1.

The cost of using a multi-sample forward ray tracing technique rather than a single sample forward or backwards technique is that the number of rays that must be computed is greatly increased, in the case of this work, by at least two orders of magnitude. To overcome this problem the forward ray tracing algorithm has been designed with the graphical processing unit (GPU) in mind and is implemented in OpenCL [18].

\section{Ray Tracing Algorithm Design}

The forward ray tracing algorithm starts with creating a set of ray starting points perpendicular to the illumination direction (in this case the sun) as shown in Figure 2. Rays are then propagated towards the spacecraft, but their direction is perturbed by up to the angular size of the illumination source (at $1 \mathrm{AU}$ the Sun diameter subtends an angle of $\simeq 0.53^{\circ}$.). The intersection of these rays with the spacecraft structure is then computed as shown in Figure 3. The intersection between a ray and an object is calculated by solving the set of equations that describe the ray and the objects surface in $3 \mathrm{D}$ space. This yields the distance of the intersection along the ray. Every object, in this case the triangles making up the surface of the Solar Orbiter model, must be tested for possible intersection with the ray. The spacecraft models used for this work were the same geometries used in PTetra in order to allow for direct comparison with the existing case. This means that each ray must be tested for intersection with many thousands of individual triangles.

In this work ray/triangle intersections are calculated using the Möller-Trumbore intersection algorithm [19]. Previous ray/triangle intersection algorithms first calculated the intersection of the ray with the plane on which the triangle lies. A
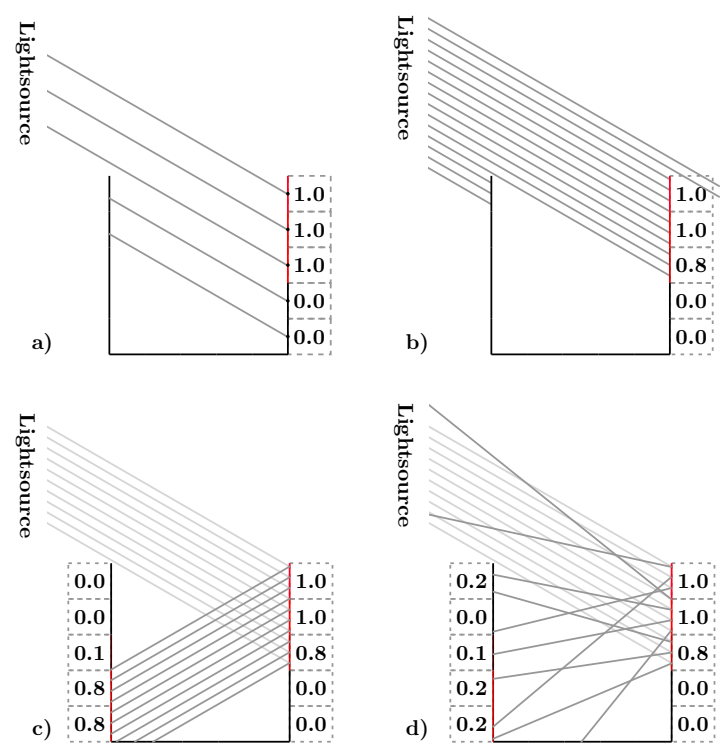

Fig. 1. Backwards ray tracing (a) propagates a single ray from each element and checks to see if it reaches the lightsource. In forward ray tracing (b) many more rays are propagated and the number of intersections is counted for each element. Specular reflection (c) and diffuse reflection (d) can both be modelled in this technique.

test was then carried out to determine if this point was inside the edges of the triangle.

The Möller-Trumbore algorithm takes a different approach. A transformation is constructed and applied to the origin of the ray. The transformation yields a vector containing the distance, $t$, along the ray to the intersection point and the barycentric coordinates, $(u, v)$, of the intersection. In this way the ray/plane intersection of previous algorithms is avoided.

A triangle is defined by three vertices $V_{0}, V_{1}$ and $V_{2}$ and a ray defined as having an origin $O$ and direction $D$. In this approach three new vectors are defined as $E_{1}=V_{1}-V_{0}$, $E_{2}=V_{2}-V_{0}$ and $T=O-V_{0}$. The intersection can be found by solving the following:

$$
\left[\begin{array}{l}
t \\
u \\
v
\end{array}\right]=\frac{1}{P \cdot E_{1}}\left[\begin{array}{c}
Q \cdot E_{2} \\
P \cdot T \\
Q \cdot D
\end{array}\right]
$$

Where $P=\left(D \times E_{2}\right)$ and $Q=\left(T \times E_{1}\right)$.

Once the intersection point, if it exists, of a given ray and the geometry has been determined a reflected ray can be generated using the triangle's surface normal. The overall reflectivity of the material is implemented by controlling the proportion of incoming rays that generate reflected rays. For example if the reflectivity of the material is 0.9 then there is a $90 \%$ chance that a reflected ray is created and a $10 \%$ chance that the ray terminates at the computed intersection point.

In the case of a purely specular reflection, the reflected ray is created by rotating the incident ray $\pi$ radians around the normal and reversing its direction. In the case of any diffuse component of the reflection another approach must be taken. The Lambert model [20] describes a perfectly diffuse surface 
that scatters the incoming photons in many directions. In the Lambert model the reflected ray direction is sampled from a distribution based on Lambert's cosine law, which states that the amount of light emitted from an ideal diffuse radiator is directly proportional to the cosine of the angle $\theta$ between the direction of the incident light and the surface normal. The Lambert model is crude but is sufficient for modelling the diffuse characteristics of materials in this study.

The new ray created by reflection is treated in exactly the same way as the original rays, and will propagate through the model space, possibly reflecting multiple times. As the reflectivity coefficient of the material is implemented by controlling the number of rays reflected, the rays themselves do not diminish as they reflect.

Once all of the ray/spacecraft intersections have been calculated, including intersections from reflected rays, the number of intersections per triangle is computed. In this way the model is quantised and a given triangle will have a integer number of rays striking it. The error introduced by this quantisation is overcome by initially generating hundreds of millions of rays. This results in each triangular face of the spacecraft receiving hundreds of rays. In this model the triangles have a side length of approximately $0.1 \mathrm{~m}$. A check is performed to determine the error generated by this quantisation. A triangle of known size, aligned perpendicular to the incoming rays will have a normalised illumination of exactly 1 . Test triangles are put into the ray tracer and their illumination determined by counting the number of intersections of rays. These test triangles produced illumination values with an error of less than $0.1 \%$ when compared to the ideal triangle.

In order to generate and propagate hundreds of millions of rays the ray tracing algorithm itself was implemented in OpenCL and designed to run on a GPU. A GPU consists of many of hundreds of small processors, designed specifically to carry out many simple parallel computations. As such they are perfectly suited to ray tracing. A ray can be propagated independently on each of the GPU's processors, meaning orders of magnitude speed improvements compared to identical algorithms on CPUs, which have a much smaller number of general purpose processors. In order to take advantage of the GPU, the algorithm was implemented in a general purpose GPU language, OpenCL. OpenCL itself makes a limited number of methods available, but these are sufficient for implementing the ray/triangle intersection method previously described.

\section{INTEGRATION WITH PTETRA}

Illumination maps generated using the algorithm described above with and without multiple reflection, are used in the particle in cell (PIC) satellite-environment simulation code PTetra. In PTetra space is discretised using an unstructured and adaptive tetrahedral mesh. One advantage of using an unstructured mesh is that it enables a good representation of a satellite with complex geometries, on which realistic boundary conditions can be applied. With this type of mesh, physical object surfaces are defined by contiguous triangles, which are faces of tetrahedra defined in the simulation domain. In

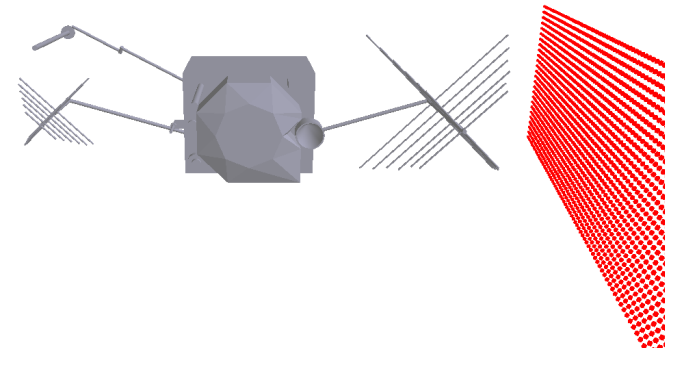

Fig. 2. An array of points is created as the source of the rays.

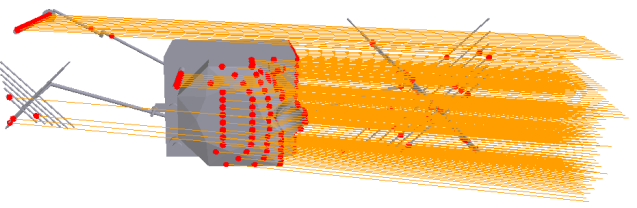

Fig. 3. These rays are propagated and intersected with the spacecraft.

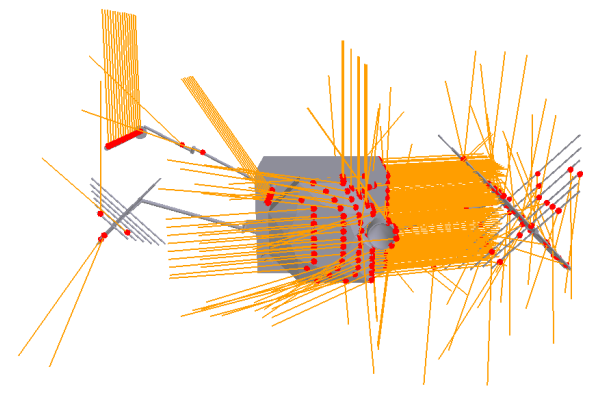

Fig. 4. Reflected rays are created based on the surface normal and material properties.

PTetra, only the region of space between satellite components, or physical objects and a user-defined outer boundary, is represented by the mesh. Volumes inside physical objects are not accounted for. A tetrahedral mesh is defined in the code from

- the list of $x, y, z$ coordinates of all vertices, and

- the list of the four vertex indices and neighbours for all tetrahedra

For example the list of coordinates would contain entries such as

$14.0-017.6-011.1+00$

$24.0-01-7.6-011.1+00$

where the first column lists the vertex indices, and the 
following three columns list the $x, y, z$ coordinates. The tetrahedral elements are then defined with entries such as

$\begin{array}{lllllllll}10 & 1831 & 3562 & 1832 & 8513 & 12 & 11159 & 590 & -2013\end{array}$

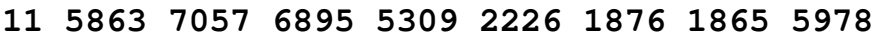

where the first column gives the element indices, the following four integers lists the four vertices making this tetrahedron, and the last four integers give the indices of the tetrahedra opposite each vertex in the same order as listed in columns 2 to 5 . Note that, in this example, one "neighbour" index is negative. Negative indices refer to physical boundary indices. This is how PTetra recognises different boundaries on which different boundary conditions can be applied. The interface between the ray tracing code and PTetra is made via a file consisting of three columns. The first column gives the list of all tetrahedral elements with a face on a physical structure. The second column gives the index of the vertex in this tetrahedron (as it appears in the mesh file) opposite the physical structure. Finally the third column gives the relative illumination of the face on a structure, as determined by the ray tracing code. For example,

$\begin{array}{lll}5361 & 4 & 1.23579732074\end{array}$

654210.977312452683

shows that in element 5361, the face opposite the fourth vertex listed for that element receives a relative illumination of 1.23579732074. Here a relative illumination of 1.0 is assigned to a surface element receiving solar radiation at normal incidence over its full area. A relative illumination larger than one corresponds to a triangular element being illuminated more than once due to multiple reflections, and an illumination lower that unity results from a triangular element being illuminated at an oblique angle or being only partly illuminated due to part of its surface being in the shade of a physical object. From the surface material and the distance to the Sun (known in PTetra), the actual solar flux and emitted photoelectron current density can then be calculated from a simple multiplicative factor.

\section{ILLUSTRATION WITH A SIMPLE "TOY MODEL"}

We first illustrate the effect of multiple reflections with a simple geometry where reflection patterns and their effect on the electron distribution function and collected current densities are clearly visible. This "toy geometry" consists of two rectangular prisms of dimension $0.4 \times 2.8 \times 2.8 \mathrm{~m}$ in $x, y, z$ respectively, with a cylinder of height $1.8 \mathrm{~m}$ and a radius of $0.4 \mathrm{~m}$ located halfway between the plates, as illustrated in Figs. 5 and 6. Simulations are made without and with multiple reflection effects assuming a background stationary plasma and the sun in the direction of $(1 ., 0.2,0.1)$ in the object's system of coordinates. When accounting for multiple reflections, we assume an albedo of 0.9 and $90 \%$ of specular reflection. In both cases an unmagnetized fully ionized hydrogen plasma of density $n_{e}=n_{i}=10^{10} \mathrm{~m}^{-3}$, and temperature $T_{e}=T_{i}=1 \mathrm{eV}$ is assumed, and photoelectron emission is calculated assuming aluminum oxide as surface material. The effect of multiple reflections is clearly visible in Figs. 5 and 6 showing the net collected current per unit surface area on different surface components, obtained in the two cases. The areas with positive collected current correspond to locations where photoelectron emission overcomes collection of background plasma electrons, since emission of electrons is equivalent to positive current collection. With direct illumination only, photoelectron emission occurs on the side of the right rectangular prism facing the sun, and on a narrow Lshaped band on the inside of the left prism. No photoelectron emission occurs on the cylinder between the two prisms or on the inner side of the right prism. With multiple reflections, however, several bands of photoelectron emission (positive collected current density) are visible on both inner faces of the rectangular prisms, as well as on the cylinder top and side. The resulting enhanced photoelectron emission between the two prisms affects plasma parameters in this region as shown in Fig. 7 for the plasma potential along the $x$ axis ( $y=0, z=0)$ computed without (solid) and with (dashed) multiple reflections. The plasma potential between the plates and the cylinder is seen to be slightly less negative when multiple reflections are taken into account. The prisms are also less negative by approximately $0.1 \mathrm{~V}$ with multiple reflections, and the cylinder by approximately $0.25 \mathrm{~V}$. These differences of 4 to $9 \%$ are indicative of variations in other plasma parameters such as the ion and electron density between the plates, associated with multiple reflections. Finally we note that the relatively small changes found here are consistent with the plasma density of $10^{10} \mathrm{~m}^{-3}$ assumed in the simulation, at which photoelectron emission plays a minor role in the net current balance in floating objects. In a lower density plasma, photoelectron emission should be more significant as the contribution of photoelectron emission to current balance should be relatively lager.

\section{USE OF TECHNIQUE FOR SOLAR ORBITER}

We now turn to PTetra simulation results obtained with photoelectron emission calculated from illumination maps provided by the ray tracing code. As a case study we consider the geometry of Solar Orbiter illustrated in Fig. 8. Assuming a distance from the Sun $R=0.6 \mathrm{AU}$, solar illumination is enhanced by a factor $\sim 2.8$ compared to what it is at Earth orbit, and the Sun angular width is approximately $\delta \theta_{\odot} \sim 0.88^{\circ}$. In the simulations a conservative flying attitude is assumed in which the satellite shield is directly facing the Sun. As a result photoelectrons are primarily emitted from the satellite components directly exposed to the Sun. These are two large solar panels and the front shield. Much lower photoelectron emission is expected from the sides of the satellite exposed to grazing radiation associated with the Sun finite angular width. Under the assumed flying attitude, the central satellite body located behind the shield, on which most scientific instruments will be located, should be exposed to no solar radiation.

\section{REsults AND Future Work}

The enhancement in photoelectron emission caused by multiple reflections is assessed by comparing results obtained from 

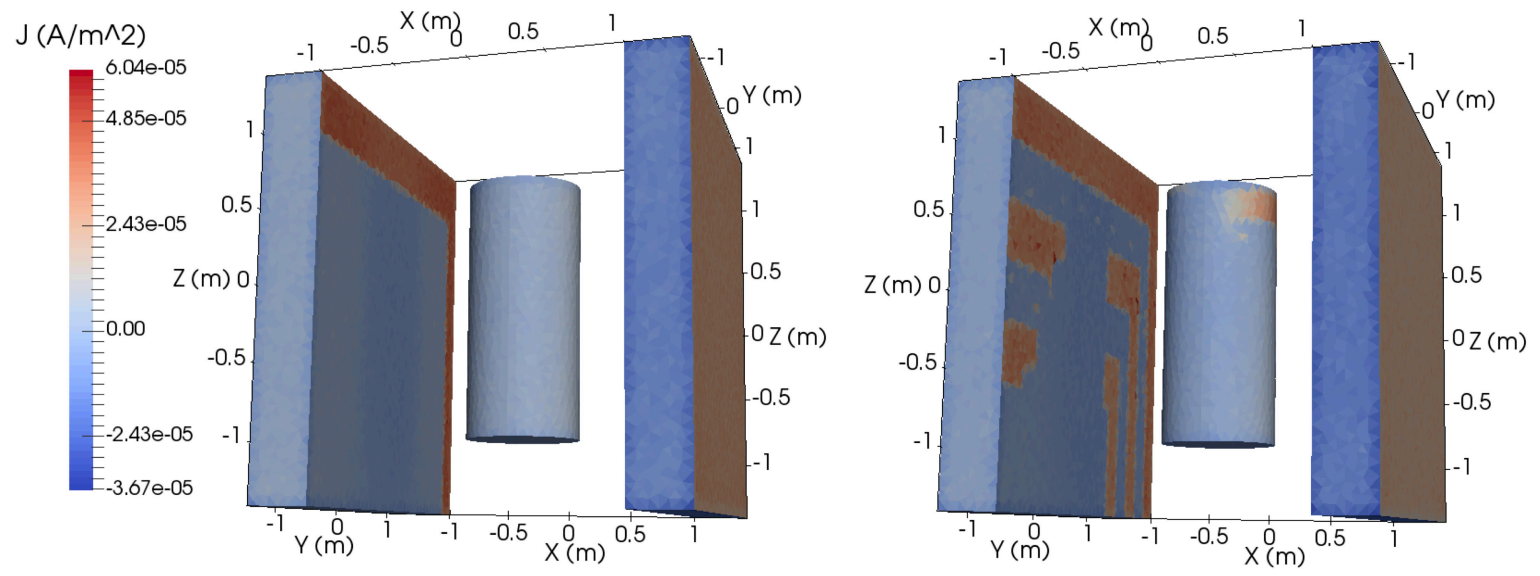

Fig. 5. Collected current density, looking at the inner face of the left prism calculated without (left) and with (right) multiple reflections. Photoelectron emission corresponds to positive current collection.
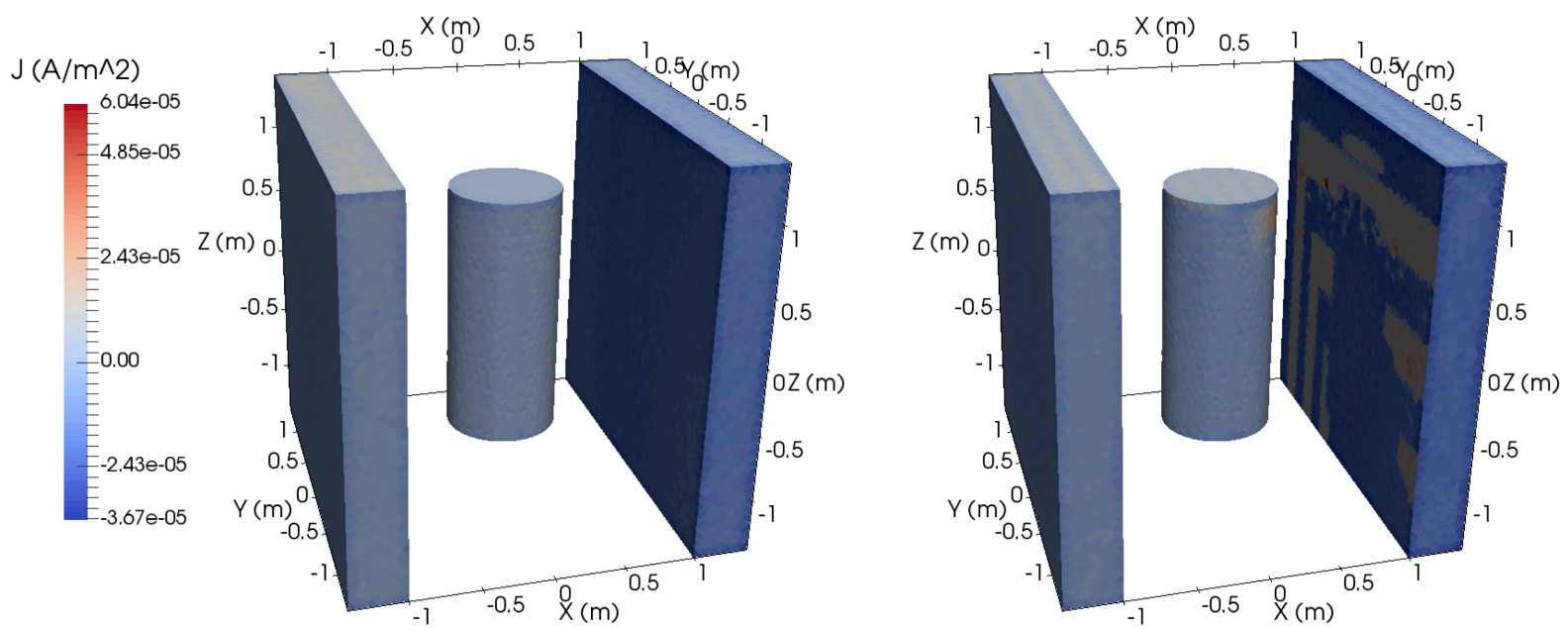

Fig. 6. Collected current density, looking at the inner face of the right prism calculated without (left) and with (right) multiple reflections. Photoelectron emission corresponds to positive current collection.

two simulations. A first case used as reference is simulated with direct illumination only; that is, without any reflection. The other is obtained with multiple reflections taken into account, assuming $50 \%$ specular reflection and $50 \%$ isotropic diffuse reflection, and an albedo of $90 \%$. The solar wind parameters assumed in the simulations are summarised in table I. In both cases simulations are carried out until the system

TABLE I

SOLAR WIND PARAMETERS ASSUMED IN THE SIMULATIONS

\begin{tabular}{|l|l|}
\hline$n_{e}=n_{i}$ & $2.42 \times 10^{7} \mathrm{~m}^{-3}$ \\
\hline$T_{e}=T_{i}$ & $10 \mathrm{eV}$ \\
\hline ion species & $100 \% \mathrm{H}^{+}$ \\
\hline solar wind speed & $4.1 \times 10^{5} \mathrm{~m} / \mathrm{s}$ \\
\hline magnetic flux density & $5 \mathrm{nT}$ \\
\hline
\end{tabular}

reaches a steady state. Cross sections of the ion and electron density, and the electric potential computed without multiple reflection are shown in Fig. 9. Profiles computed with multiple reflection effects are very nearly the same, and are not shown here. With multiple reflections parts of the back side of the satellite are exposed to indirect solar radiation, and emit a small but finite level of photoelectrons.

Figure 10 shows profiles of the net current density collected on the back side of the satellite, calculated without (top) and with (bottom) multiple reflections. Collected solar wind electrons and photoelectrons contribute a negative current density, while the emission of photoelectrons on a surface contributes a positive current density. The contribution from ions to the collected current density is negligible on the back side of the spacecraft, owing to the supersonic speed of the solar wind. Without multiple reflections (top panel) the back side of the satellite collects solar wind electrons, and photoelectrons emitted from other parts of the satellite, which results in a negative collected current everywhere on that face. 


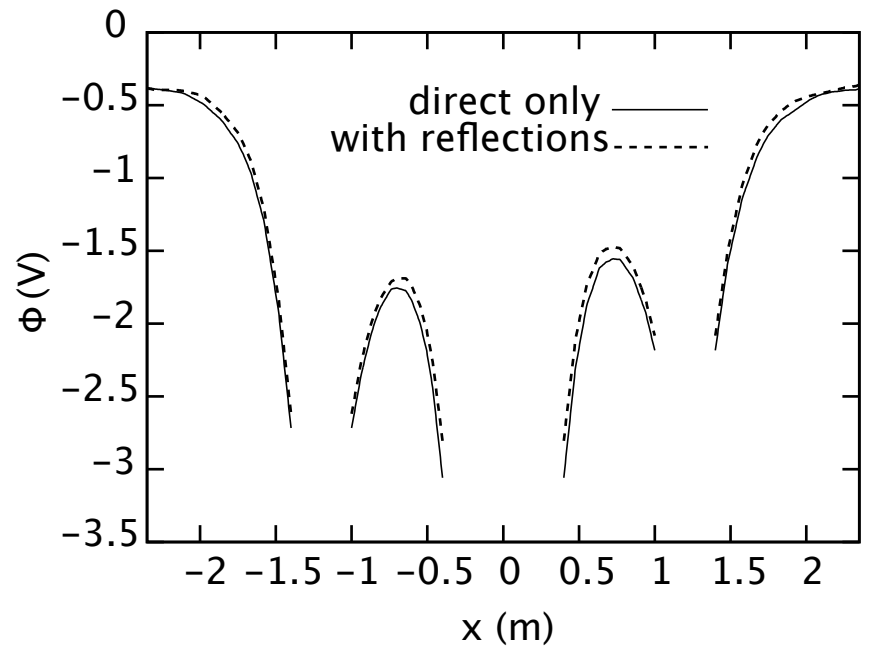

Fig. 7. Potential along the $x$ axis $(y=0, z=0)$ computed without (solid) and with (dashed) multiple reflections.

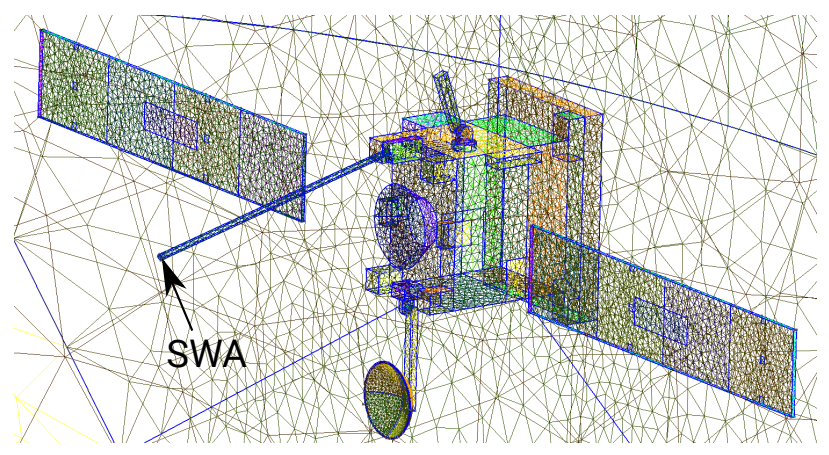

Fig. 8. Illustration of the Solar Orbiter geometry used in the simulations. The mesh resolution of the outer boundary has been coarsened in order for the spacecraft to be more visible.

With multiple reflections (lower panel) however, parts of the back side now emit photoelectrons, which tends to lower the net collection of electron current. At some locations, emitted photoelectrons are seen to exceed the collection of ambient electrons, which leads to a net positive collected current per unit surface area. The regions where the current density is visibly less negative or positive are distributed irregularly on the back side of the main satellite body. These patches are due to reflections on the sun-exposed convex face of the parabolic antenna, which is represented in the simulations, with an assembly of planar triangular faces. With a smooth surface for the sun-exposed face of the antenna, reflected light would be distributed smoothly on the back side of the satellite, and the resulting photoelectron emission and associate (positive) collected current density would also exhibit a smooth profile.

Owing to the relatively small surface area affected by multiple reflection, and the positive floating potential of the spacecraft, it is found in this case that plasma parameters surrounding the spacecraft, are very nearly the same when computed with or without reflection effects. For example, the difference between spacecraft floating potentials obtained in the two cases is of order $0.5 \%$. It is difficult to assess the uncer-
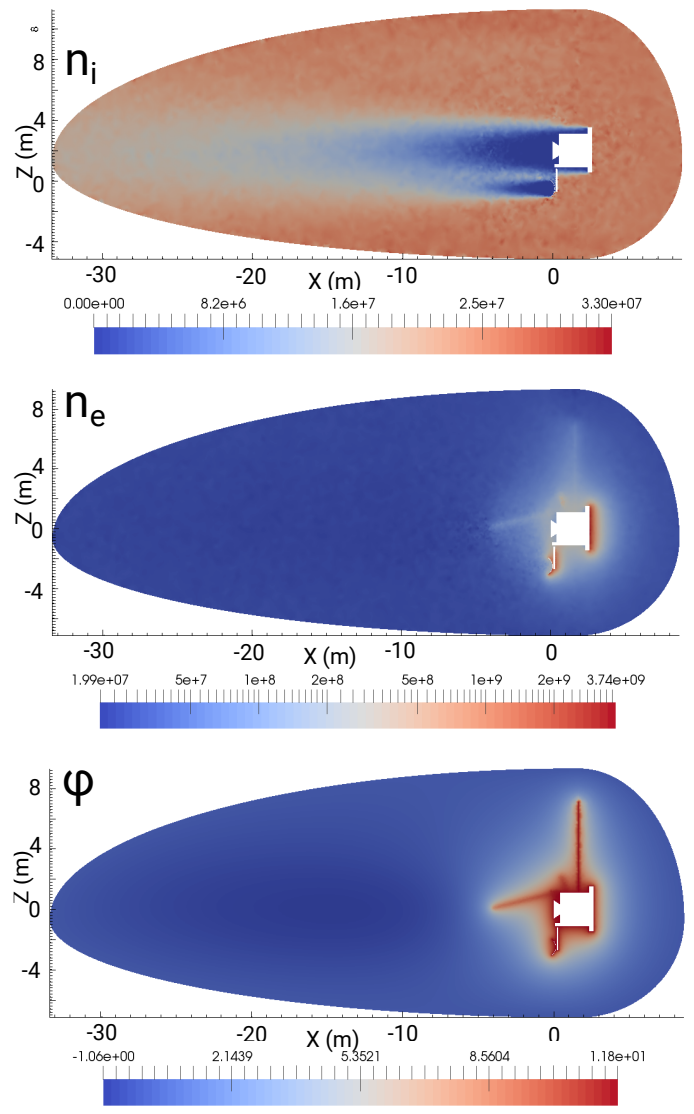

Fig. 9. Cross section of the ion density (top), electron density (middle) and electric potential (bottom) in the $x-z$ plane, computed with direct solar illumination only. Profiles computed with multiple reflections in this case are essentially the same as the ones shown in the figure.

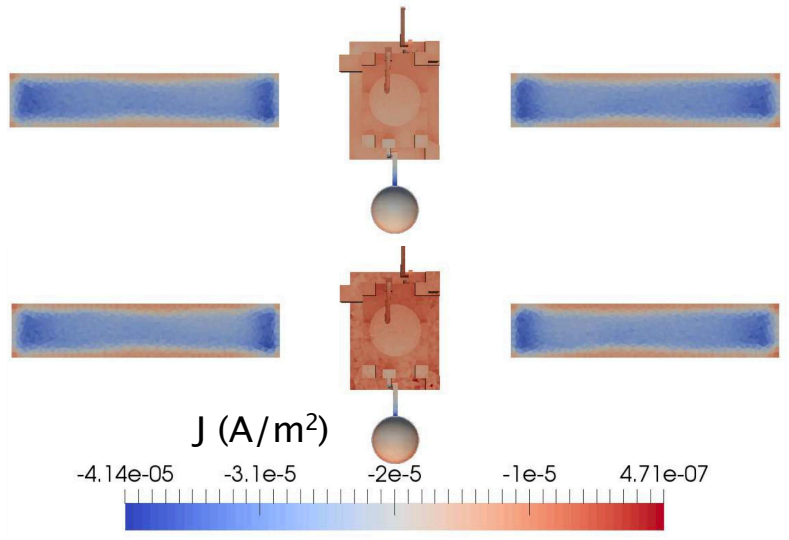

Fig. 10. Net current density collected at the rear of the satellite when emitted photoelectrons are calculated with direct solar illumination only (top) and with multiple reflections (bottom).

tainty in the computed floating potential, which is affected by a number of factors including an approximate representation of the spacecraft geometry, the size of the simulation domain, the assumed boundary conditions, and discretisation errors in the numerical solution of Poisson's equation. It is likely nonetheless that the small difference found for the floating potential and surrounding plasma parameters are within the 
error bars in these quantities. One location of particular interest with Solar Orbiter, is the tip of the boom located in the wake of the satellite body, were the Solar Wind Analyzer (SWA) will be located. Electron distribution functions have been calculated at that location, and again practically no differences were found between simulations results obtained with or without multiple reflection effects.

\section{SUMMARY AND CONCLUSION}

A computer model has been developed to compute solar illumination of satellite components while accounting for multiple reflections, variable surface properties and illumination source size. The code can optionally trace reflected rays assuming an arbitrary combination of specular and diffuse reflection. Modules have been written to read 3D unstructured tetrahedral geometry as used in the PIC code PTetra, and produce illumination maps directly read in PTetra thus enabling kinetic simulations in which photoelectron emission resulting from direct illumination and multiple reflections are taken into account. Similarly modules could readily be written to produce illumination maps readable by any other satellite-environment simulation code.

Simulations made for a simple "toy geometry" show that the inclusion of multiple reflections in the calculation of solar illumination and associated photoelectron emission, can lead to differences in collected current density patterns on surface components, and on plasma macroscopic parameters. While the relatively high density $\left(10^{10} \mathrm{~m}^{-3}\right)$ case considered led to small variations, multiple reflection effects would likely be more important in lower density plasmas due to the fact that in such cases, emitted photoelectrons should contribute more in the current balance to material components. The simulations made for the Solar Orbiter geometry show that, under assumed solar wind conditions and orbital position, macroscopic parameters such as the density or space charge potential are for practical purposes, unaffected by multiple reflections. In particular, the electron distribution near SWA is found to be unaffected by photoelectron emission resulting from reflected solar radiation on the back side of the spacecraft. This is due in part to the small area exposed to this reflected radiation, and also in part because of the positive floating potential of the spacecraft resulting from the solar wind conditions considered.

Multiple reflections should be more significant however, in low density solar wind plasma when a heat shield is not required, and where spacecraft or lander components would be directly exposed to solar radiation. The next step in this analysis should consist of simulating the environment of Solar Orbiter and other satellites assuming various flying attitudes and space weather conditions, in order to better assess multiple reflection effects on particle sensor measurements.

\section{ACKNOWLEDGMENT}

S Grey would like to thank the Department of Civil, Environmental and Geomatic Engineering at University College London, and the Department of Mechanical and Aerospace Engineering at the University of Strathclyde for all of their support.
R Marchand acknowledges financial support from the Natural Sciences and Engineering Council of Canada.

\section{REFERENCES}

[1] Ira Katz, Myron Mandell, Gary Jongeward, and M. S. Gussenhoven. The importance of accurate secondary electron yields in modeling spacecraft charging. Journal of Geophysical Research: Space Physics, 91(A12):13739-13744, 1986.

[2] R. Schmidt and A. Pedersen. Long-term behaviour of photo-electron emission from the electric field double probe sensors on GEOS-2. Planetary and Space Science, 35(1):61 - 70, 1987.

[3] S.T. Lai. Fundamentals of Spacecraft Charging: Spacecraft Interactions with Space Plasmas. Princeton University Press, 2011.

[4] H. E. Hinteregger, K. R. Damon, and L. A. Hall. Analysis of photoelectrons from solar extreme ultraviolet. Journal of Geophysical Research, 64(8):961-969, 1959.

[5] E. C. Whipple. Potentials of surfaces in space. Reports on Progress in Physics, 44(11):1197, 1981.

[6] Anders I. Eriksson and Erik Winkler. Photoemission current and solar EUV radiation: Cluster and TIMED observations. In Proceedings of the 10th Spacecraft Charging Technology Conference, 2007.

[7] Dale C. Ferguson. New Frontiers in Spacecraft Charging. IEEE Transactions on Plasma Science, 40(2):139-143, February 2012.

[8] The Solar Orbiter Team, D. Mller, R. G. Marsden, O. C. St. Cyr, and H. R. Gilbert. Solar Orbiter: Exploring the SunHeliosphere Connection. Solar Physics, 285(1-2):25-70, July 2013.

[9] S. Guillemant, J. C. Mato-Vlez, V. Gnot, P. Sarrailh, A. Hilgers, and $\mathrm{Ph}$ Louarn. Study and simulation of low energy plasma measurement on solar orbiter. In Spacecraft Charging Technology Conference 2014 (13th SCTC), 2014.

[10] Richard Marchand. PTetra, a Tool to Simulate Low Orbit SatellitePlasma Interaction. IEEE Transactions on Plasma Science, 40(2):217229, February 2012.

[11] Shu T. Lai. Importance of Surface Conditions for Spacecraft Charging. Journal of Spacecraft and Rockets, 47(4):634-638, July 2010.

[12] R. E. Davies and J. R. Dennison. Evolution of secondary electron emission characteristics of spacecraft surfaces: Importance to spacecraft charging. 1998.

[13] Maurice Tautz. Analytic models for a rapidly spinning spherical satellite charging in sunlight. Journal of Geophysical Research, 110(A7), 2005.

[14] S.T. Lai and M.F. Tautz. Aspects of Spacecraft Charging in Sunlight. IEEE Transactions on Plasma Science, 34(5):2053-2061, October 2006.

[15] Benoit Thiebault, Benjamin Jeanty-Ruard, Pierre Souquet, Julien Forest, Jean-Charles Mateo-Velez, Pierre Sarrailh, David Rodgers, Alain Hilgers, Fabrice Cipriani, Denis Payan, and Nicolas Balcon. SPIS 5.1: An Innovative Approach for Spacecraft Plasma Modeling. IEEE Transactions on Plasma Science, 43(9):2782-2788, September 2015.

[16] Marek Ziebart. Generalized analytical solar radiation pressure modeling algorithm for spacecraft of complex shape. Journal of spacecraft and rockets, 41(5):840-848, 2004.

[17] M. Ziebart, S. Adhya, A. Sibthorpe, S. Edwards, and P. Cross. Combined radiation pressure and thermal modelling of complex satellites: Algorithms and on-orbit tests. Advances in Space Research, 36(3):424-430, January 2005.

[18] John E Stone, David Gohara, and Guochun Shi. Opencl: A parallel programming standard for heterogeneous computing systems. Computing in science \& engineering, 12(1-3):66-73, 2010.

[19] Tomas Mller and Ben Trumbore. Fast, Minimum Storage Ray/Triangle Intersection. In ACM SIGGRAPH 2005 Courses, SIGGRAPH '05, New York, NY, USA, 2005. ACM.

[20] J.H Lambert. Photometria sive de mensura et gradibus luminis, colorum et umbrae. Eberhard Klett: Augsberg, Germany, 1760. 

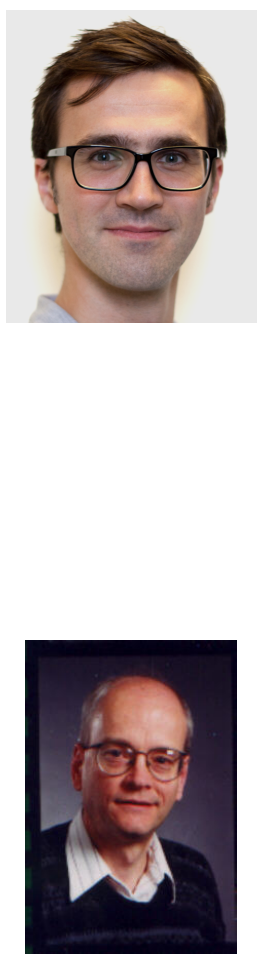

Richard Marchand received his $\mathrm{PhD}$ in Astrophysics from Princeton University. After graduating his research interests included tokamak microinstabilities and transport, laser-plasma interactions, and space plasmas. His current research focus is on developing and using computer models to simulate the interaction of space environment with satellites.

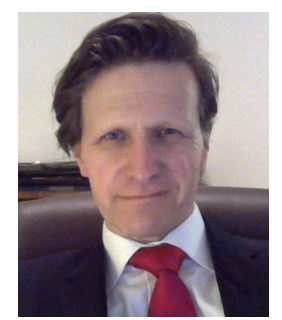

Marek Ziebart is Professor of Space Geodesy at University College London. His principal research area is the modelling of non-conservative forces on space vehicles. His group has developed models used as operational standards by NASA, and that are used in the precise orbit determination and prediction of GPS satellites for scientific applications.

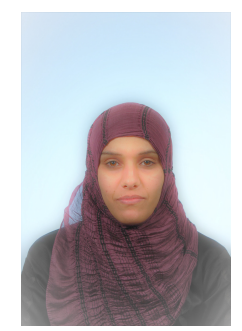

Stuart Grey received the MEng degree in Aerospace Engineering from the University of Sheffield, UK, in
2004 and the Ph.D. degree in Aerospace Engineering from the University of Glasgow, UK, in 2012. He of Strathclyde. His current research interests are spacecraft charging, spacecraft force models and numerical modelling. 